\title{
Senor-based Personal Protective Equipment in forestry work with dangerous machines and equipment (power saws) - IGF No. $16119 \mathrm{~N}$
}

\author{
Dr. Jan Beringer; Angela Mahr-Erhardt, Hohenstein Institut Hohenstein Institut für \\ Textilinnovation gGmbH, Deutschland, j.beringer@hohenstein.de, a.mahr-erhardt@hohenstein.de \\ Dr. Peter Hoffmann, TZI Bremen University, Bremen, phoff@tzi.de
}

\begin{abstract}
:
Forestry work is an activity with one of the highest injury and accident hazard. Besides the professional use power saws are more and more used in the private sector, this mainly because of increasing energy costs. The aim of the work is the development of an intelligent sensory protective system which will make the work with power saws less dangerous. If the power saw comes closer than some centimetre to the body, the sensory active cut protection trousers will stop the power saw. This will be reached by the integration of sensors into the trousers which are able to detect the distance and position of the chain guide and activate the emergency stop of the power saw.
\end{abstract}

Key words: Personal Protective Equipment (PPE), forestry work, sensor-active protective layer, detection of magnetic fields, inductive proximity switches

The continually increasing use of power saws, for example in the processing of firewood, leads to an increasing demand for related protective equipment. In Germany alone, there are about 25,000 people working professionally in forestry, about half of them in privately-owned woodland. In addition, there are persons, who carry out tree felling and cutting work during the maintaining of private gardens and public parks. Rising prices for fossil fuels lead to a strong increase of private users, who process their firewood with a power saw themselves. This involves a high risk potential, which can be addressed by the developments in this project.

The Personal Protective Equipment (PPE) of the forestry workers comprises special protective clothing, which to date only provides passive cut protection. The research project contributes to the safe application of power saws through an innovative further development of the existing safety equipment by an active electronic component.
In comparison to passive cut protection, electronic systems offer the advantage of an early detection of dangerous situations. The early generation of a switch-off signal considerably minimizes both, material losses and the risk of physical injuries.

The project supplements the passive protection offered by cut-protective trousers through the development of active proximity detection and the generation of a switch-off signal, as soon as a cutting damage is imminent. This has been implemented by means of a sensor-active textile layer by applying proximity sensor technology between power saw and textile.

The project examined the principle of the detection of magnetic fields with regard to a possible application in forestry work. In this connection, the proximity detection is extremely critical in terms of distance and time. A user survey revealed that for $80 \%$ of the interviewees, the minimum distance between the guide rail and the foot frequently lies between 5.0 and $10.0 \mathrm{~cm}$. The guide rail of the power saw is a 
wearing part, which is replaced several times a year in the case of professional workers. In terms of retrofit solutions regarding the generation of the required magnetic field, an integration of permanent magnets into the guide rail is especially suitable.

From the automation technology sector, inductive proximity switches are known. The microsystems technology has made enormous progress regarding magnetic field sensors over the last years. The research project was implemented using low-cost and robust Reed switches. These Reed switches can be operated without quiescent power consumption and are thus the most economical solution for mobile textile applications.

For the adaption of the Reed switch and for the composition of the sensor matrix a conductive textile carrier material was used.

This sensor-active textile is being introduced between the cut-protective layers and the exterior layer during making up and is not visible. Practical stress tests at the Hohenstein Institute have shown that the solution is very robust against mechanical impact and withstands several care cycles safely.

A further advantage of the solution is that the sensor-active protective layer does not have to be removed during washing - it can remain in the piece of garment. The composition of the textile sensor layer does not impose any limitations during making up. It can be handled without any major restrictions in an industrial manufacturing process. It is thus particularly well suited for the integration in a wide variety of protection equipment for further applications which are involving a high risk potential.

The IGF project 16119 / N Research Association Textile Research Council eV, Reinhardt Strasse 12 - 14, 10117 Berlin was the AIF under the program for the promotion of industrial research and development (IGF) by the Federal Ministry of Economics and Technology based on a decision of the German Bundestag encouraged.

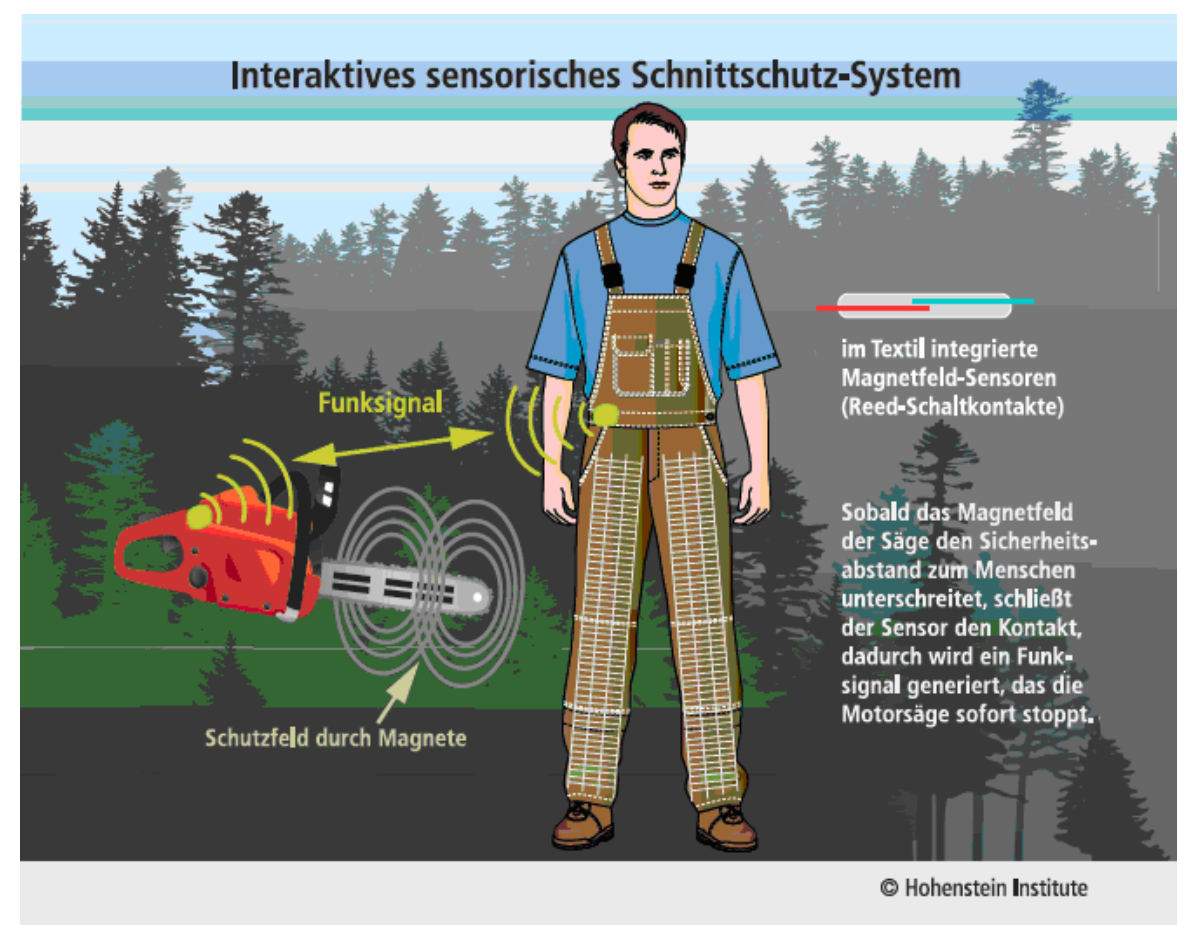

Abb.1: Schematic diagram of the experimental setup protective trousers, Chainsaw and proximity detection 